\title{
Direct Investment Casting For Pattern Developed By Desktop 3D Printer
}

\author{
O.M.F. Marwah ${ }^{1,3, *}$, M.S. Shukri1 ${ }^{1,3}$, E.J. Mohamad $^{2}$, M.A. Johar ${ }^{1}$, R.H.A. $\mathrm{Haq}^{1,3}$, R.K. \\ Khirotdin ${ }^{1,3}$ \\ ${ }^{1}$ Department of Manufacturing and Industrial Engineering, Faculty of Mechanical and Manufacturing \\ Engineering, Universiti Tun Hussein Onn Malaysia, 86400 Johor, Malaysia \\ ${ }^{2}$ Department of Mechatronics and Robotics Engineering, Faculty of Electrical and Electronic \\ Engineering, Universiti Tun Hussein Onn Malaysia, 86400 Johor, Malaysia \\ ${ }^{3}$ Additive Manufacturing Research Group, Faculty of Mechanical and Manufacturing Engineering, \\ Universiti Tun Hussein Onn Malaysia, 86400 Johor, Malaysia
}

\begin{abstract}
Development of RP technologies has encouraged rapid study on portable 3D Printer in which there are varieties of portable 3D printer machines in market. Nevertheless, less reports regarding its consumption of fabricated pattern to be used in direct investment casting. This study has focused on development of ABS P400 pattern in terms of collapsibility behaviour which has capability to be used as sacrificial pattern in direct investment casting. In addition, the internal built structures have been built into two designs such as square and polygon patterns respectively. The patterns were constructed in semi cylindrical geometry which comes with one side opened and one side closed together wrapped with $4 \mathrm{~mm}$ of ceramic shell. This experimental were conducted with variation temperature starting from $30^{\circ} \mathrm{C}$ until $150^{\circ} \mathrm{C}$ with increment of $5^{\circ} \mathrm{C}$ per minutes while for the numerical simulation, the temperature selected was between $30{ }^{\circ} \mathrm{C}$ to $120{ }^{\circ} \mathrm{C}$ with $10{ }^{\circ} \mathrm{C}$ increment per minutes. Moreover, the observation was made that glass transition temperature, $\mathrm{Tg}$ happened near $110^{\circ} \mathrm{C}$. It was observed that the shell cracking happened on the ceramic shell. Other than that, the polygon pattern tends to collapsed inwardly rather than square pattern during the burnout process. A result also shows that, there is significant amount of stress reduction on both square and polygon which was $45 \%$ respectively. Besides that, the amount of strain on pattern itself has shown about $9 \%$ reduction. Moreover, there is greater difference in terms of ceramic shell strain reduction which was $38 \%$ for square and polygon patterns respectively. Lastly, there is $11 \%$ reduction of strain when compared square and polygon in terms of axial strain on ceramic shell
\end{abstract}

\footnotetext{
*Corresponding author: mdfaizan@uthm.edu.my
} 


\section{Introduction}

The perception of RP to be implemented as sacrificial master pattern in Investment Casting (IC) was dated back in 1989 [1]. Initially, the block mould was used in IC, and the concept is to build the RP parts that required no additional finishing. With the innovation of $\mathrm{RP}$ to be implemented in IC process, the hard tooling can be eliminated. Concurrent phenomenon, the application of RP in IC was driven by prospects of reducing costs and manufacturing times as well as assuring high quality products [2] .

Several RP machine have been developed since last 10 years such as Stereolithography (SL), Selective Laser Sintering (SLS), Laminated Object Manufacturing (LOM), Three Dimensional Printing (3DP), Fused Deposition Modeling (FDM) and Multijetmodeling (MJM) $[3-4,11]$. Due to its capability, RP techniques have emerged at the leading edge in the casting process for manufacturing of intricate parts whereby better dimensional accuracy and fine surface were requisite. Intensification of RP technique currently pays attention to desktop 3D Printer machine which similar to FDM since considered as the cheapest in the market. Nevertheless, less reports regarding the pattern printed by the desktop 3D printer as well as its capability to be used as a sacrificial pattern in IC to generate metal cast parts. This study focused on the stress developed by the portable FDM pattern which act as a direct sacrificial pattern in the IC process for fabrication of metal parts whereby, temperature and stress are the main keys to be watched.

During the burnout process, the shell will acts as stress towards pattern due to the expansion of the pattern when heat is applied. Therefore, the shell cracking occurs when the stress-strain of pattern is greater than shell resistant [5]. The consequence to this phenomenon is thicker shell induced greater shell stresses towards pattern. In addition, another criteria which is vital in determining the collapsibility of the pattern itself is the web build internal pattern structure. If the shell rupture temperature is lower than the pattern buckling temperature and the resin glass transition temperature, the cracking of the ceramic shell will occurs [6-7]. Besides that, the glass transition temperature (Tg) and Modulus of Rupture (MOR) is a relationship that significant for every polymer material when heat is presence. By using polymer materials as sacrificial pattern, the $\mathrm{Tg}$ is a reference to understand how the shell cracking occurs during the burnout process [6]. There will be a certain point of temperature when the pattern expands vigorously and reached above Tg with stress is above than MOR of ceramic shell, the shell will cracks and failed. This occurrence is related to prediction of cracking in the ceramic shell.

Acrylonitrile butadiene styrene (ABS) is a thermoplastic group in a combination of monomers of Acrylonitrile, Butadiene and Styrene. Besides that, ABS basic molecule consists of three plastic to form composite group characteristics. The wide range of using ABS materials as sacrificial pattern has been attracted reseacher due to the high impact strength, good dimensional stability, good chemical resistance, ability to prevent the flow of electricity and easily to process [10]. Besides that, ABS has a mechanical properties that are strong and hard even at temperatures as low as $-40^{\circ} \mathrm{C}$. Moreover, it also has a high thermal resistance even at fluctuated temperatures as well as having the glass transition temperature (Tg) which is $110^{\circ} \mathrm{C}$. Therefore, further investigations were undertaken by using ABS materials as a sacrificial patterns in IC process by using desktop Odyssey X2 3D Printer. 


\section{Internal RP Pattern Structure Development}

A new internal structure for investment casting(IC) for desktop 3D Printer was designed and compared between one open and one closed half cylindrical shape. Firstly, the new internal structure pattern was design with square $90^{\circ}$ and polygon styles. Fig. 1 (a) \& (b) shown the square and polygon model structures open ended. This built styles has been chosen for easier the examination of collapsibility behavior during the burnout processes. The model built with two different web structures to ensure which of both structures has tendency to collapse inwardly during the burnout process if and only if the ABS P400 does not expanding further after the glass region temperature (Tg). The internal structures represented for the ABS P400 (blue) and ceramic shell (yellow) were constructed with diameter thickness of the internal structure was $1 \mathrm{~mm}$, having height of the cylinder which was $60 \mathrm{~mm}$ and the inner diameter which was $50 \mathrm{~mm}$.

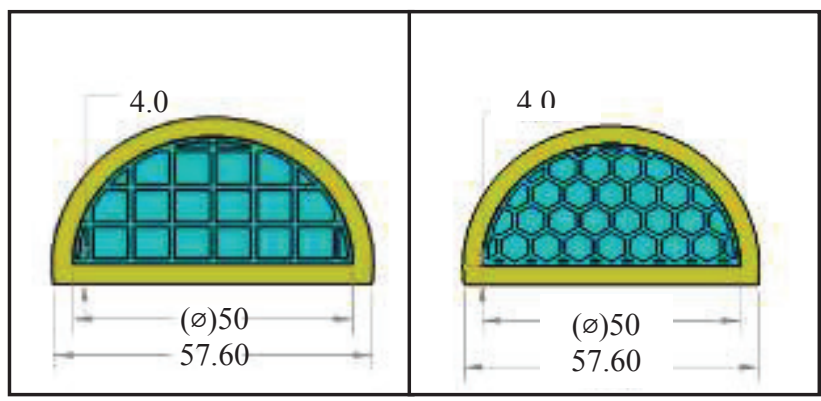

a) Square $\quad$ b) Polygon

Fig. 1. (a) Square and (b) polygon interanal web pattern structure.

This two types of internal patterns in which square and polygon built style that have one opened ended and one closed together wrapped with the $4 \mathrm{~mm}$ of ceramic shell thickness. This constructed style was different from the previous Quickcast 2.0 for external layout in which in this paper it was built with half cylinder compared to the full cylinder style. The simpler built of the pattern is to ensure that flatness of the pattern can be observed on the plain surface rather than curved surface. This constructed style was different from the previous Quickcast 2.0 for external layout in which in this paper it was built with half cylinder compared to the full cylinder style. The simpler built of the pattern is to ensure that flatness of the pattern can be observed on the plain surface rather than curved surface.

\subsection{Application of strain gauge}

For the experimental parts, the usage of strain gauge is vital due to it's ability to detect the presence of strain changed due to external pressure and temperature in the furnace. The cylindrical geometry of ceramic shell makes it possible to assume it as a thick cylinder. The difference between a thin cylinder and a thick cylinder is that a thick cylinder has a stress in the radial direction as well as a circumferential stress and longitudinal stress [8]. Fig. 2 shown that the location of strain gauge was installed on the pattern itself which are square and polygon internal web structure. 


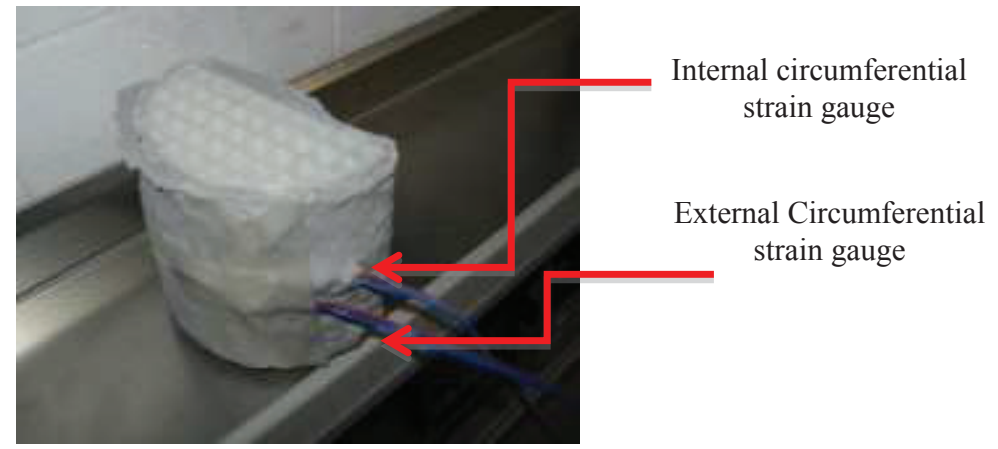

Fig. 3. The location of strain gauge attached on pattern for both square and polygon internal web structure.

In addition, radial stress has only a radial crippling effect on the wall of the ceramic shell and it is not the main criteria for defining the failure of a thick cylinder. For square and polygon models, strain gauges were installed in longitudinal and circumferential directions. Therefore, the magnitude of the strains in both directions is the comparative criteria for two structures. Besides that, the strain gauge also were attached on the ceramic shell for analyzing the strain changed on the shell surface. Furthermore, it is important to detect the strain changed on the shell due to cracking behavior of the ceramic shell when subjected to high stress and temperature.. The testing sample was placed in the controlled temperature furnace for ensuring that the temperature can be adjusted within time. The strain gauge was used with the help of data logger for collecting the responded data. Due to the sensivity of the strain gauge towards pressure and temperature, the data was taken every for $5^{\circ} \mathrm{C}$ per minutes increment starting from $30^{\circ} \mathrm{C}$ until $150^{\circ} \mathrm{C}$.

\section{Experimental Results}

\subsection{Pattern buckling of ABS P400}

To understand better about the collapsibility behaviour of ABS pattern which cause the ceramic shell to cracks, many study have been conducted. Most of the previous study have been achieved through Finite Element (FE) analysis. Nevertheless, due to the 3D geometry of the square and polygon network it was able to see the weaker point inside structures [9]. Fig. 4 shown that the patterns were undergoes burnout process and buckling happened.

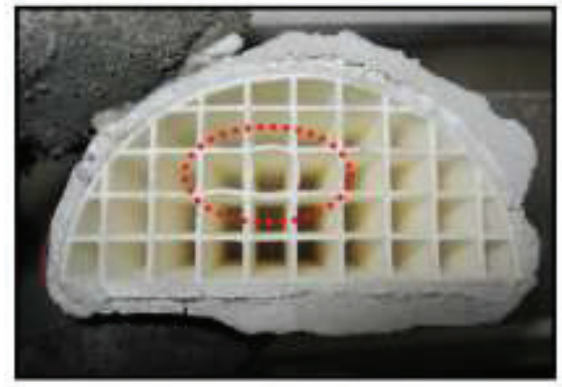

a) Square

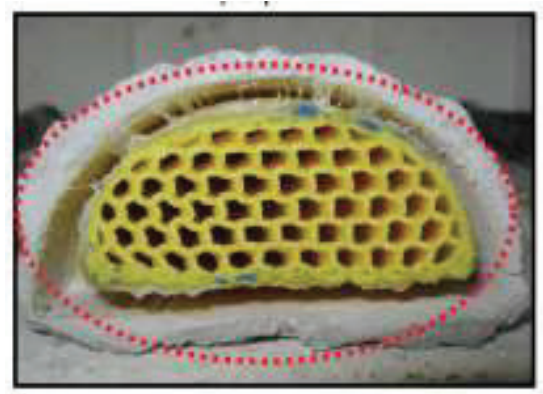

b) Polygon 
Fig. 4. Buckling of pattern during burnout process.

Half cylindrical models were used to compare the behavior of the structures during the burnout stage. The shell tends to have more strength against outer pressures; therefore, the results of this model could be applicable for other forms of 3D Printer patterns. Since the material properties of ABS P400 changes with temperature during the burnout stage, it is essential to have the diagrams of changing the mechanical properties during the heating.

This occurrence is to review the collapsibility behavior of patterns when exposed to high temperature and pressure in the furnace. It can be seen that the polygon pattern is collapsed inward rather than outward for the open ended highlighted in the red section. While for the square internal web pattern, the buckling process does not fully takes place. It can be seen that, the red section buckling only at the middle part whereby, at the side none buckling happened. When an investment casting shell with a 3D Printer epoxy pattern inside furnace oven during the burnout process, it is subjected to high temperature rise, thermal expansion, and large strains. Since the difference of the CTEs of pattern and investment ceramic is more than one order of magnitude, the pattern can exert considerable stress on the ceramic shell.

\subsection{Maximum Stress of ABS P400 Structures}

As shown at Fig. 5, the square pattern intends to received more stress rather than polygon pattern during the burnout process due to the larger difference. For the square pattern, the maximum stress induced was $38.46 \mathrm{MPa}$ while for the polygon pattern was $21.23 \mathrm{MPa}$. There was about $45 \%$ of reduction stress when compared polygon and square patterns. Results also shows the glass transition temperature for square pattern was $100^{\circ} \mathrm{C}$ while for the polygon stated that the glass trasition temperature was $90{ }^{\circ} \mathrm{C}$. Hence, based on the selected Modulus of Rupture (MOR) which was $30 \mathrm{Mpa}$ [8], square polygon undergoes cracking of shell when subjected to high thermal stress and temperature. This phenomenon happened due to the higher stability of square pattern which was not collapsing during burnout process.

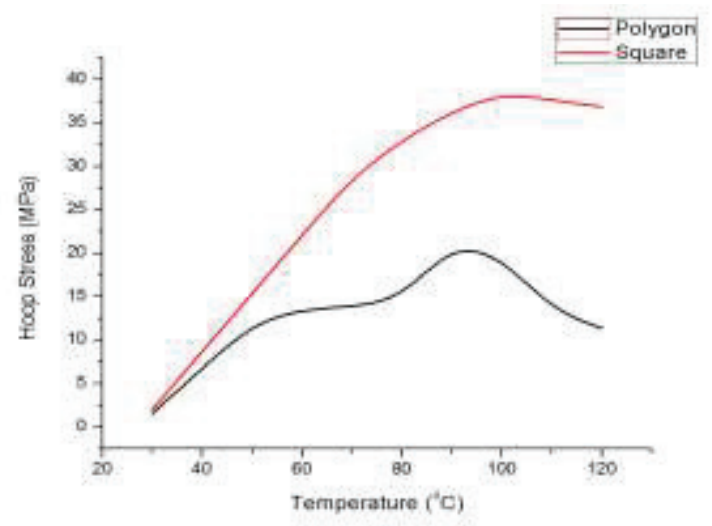

Fig. 5. Maximum Stress against Temperature

\subsection{Equivalent Strain Analysis}

According to Fig. 6(a) result shows that collapsibility behavior, polygon patterns tends to collapse easier compared to square pattern. This phenomenon shows that, square pattern 
tends to experience more strain changes compared to polygon pattern. This is due to the inner built structure of both types of ABS pattern. For the square, the design built was $90^{\circ}$ internal shape along together with supports which enable it to collapsed easier. Nevertheless, during the experimental process, the square pattern less collapsed eventhough it passed glass region temperature ( $\mathrm{Tg}$ ). It continued to gain stress from the expansion of pattern from the rise of temperature of $30^{\circ} \mathrm{C}$ until $130^{\circ} \mathrm{C}$. The strain only started to reduce when passed the temperature of $130^{\circ} \mathrm{C}$ until $150^{\circ} \mathrm{C}$. The maximum strain induced for the square pattern was 2806 microstrain. This shows that, the square pattern is hardly collapsed due to its strong build structure. According to the principle of CTE, during this phase, the pattern is most likely undergoes phase changed from solid to rubbery like. There is a limits when the maximum of temperature can be achieved by the materials pattern before it collapsed [10]. For the polygon pattern, the maximum strain induced was 2552 microstrain. There is about $9 \%$ reduction of strain when compared both square and polygon patterns. Meanwhile the polygon pattern tends to expands from beginning of $30{ }^{\circ} \mathrm{C}$ before it reached $110^{0} \mathrm{C}$ which is before the glass transition temperature $(\mathrm{Tg})$. This phenomenon can be acceptable for most of the resin or polymer material whereby, the pattern should be collapsed before the Tg.

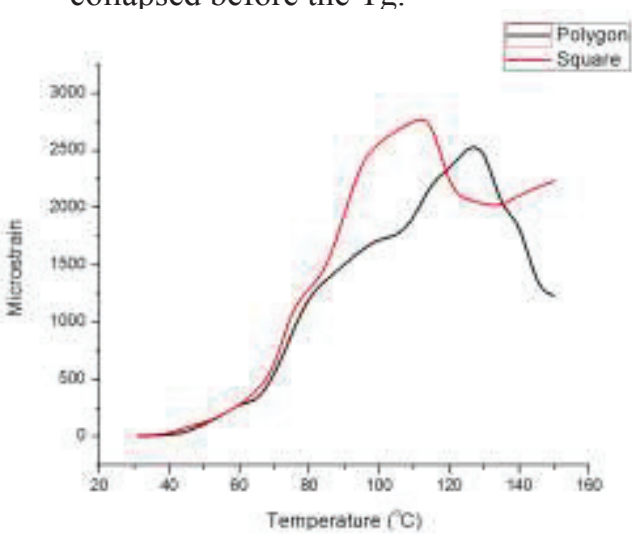

(a) Internal

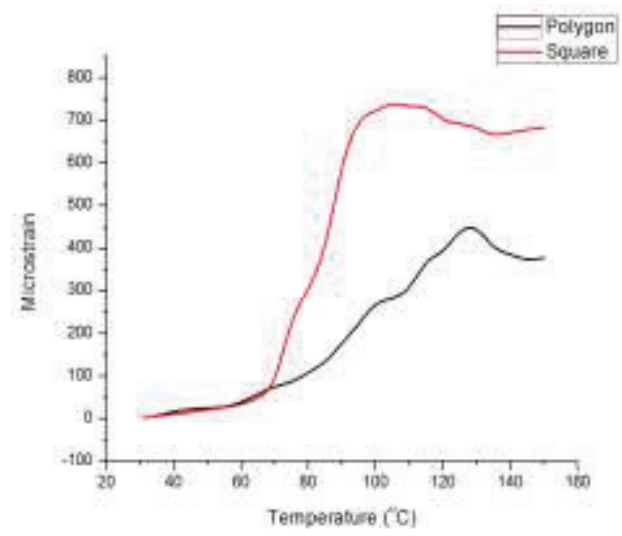

(b)External

Fig. 6. Circumferential Internal and External Circumferential Strain

From the Fig. 6(b) shown, it was stated that, square shell received more amout of strain when temperature was increased. The highest increased of temperature for square shell happened between temperature of $60{ }^{\circ} \mathrm{C}$ until $100{ }^{\circ} \mathrm{C}$. During this phase, the shell tends to achieve it's ultimate stress due to pressure contact between the pattern and shell in which the pattern expanded as well as shell too. Apart from that, however, for polygon shell it tends to yield a little bit stress on ceramic shell. Most of all, the strain was slowly increased within time. Neverthelss, it remaining to received more strain during the burnout process. Up reaching $130{ }^{\circ} \mathrm{C}$, the shell still received strain, but still less compared to the square pattern shell. This occurrence is happened due to the stronger shell to withstand the pressure expanding from the polygon pattern. It was said that, the Modulus of Rupture (MOR) of the shell must be greater than the expandeable material pattern to avoid the shell cracking during the burnout process [8]. 


\section{Conclusion}

Desktop 3D Printer ABS P400 material has validated it's ability to be used in IC process. In terms of strain on patterns there was $9 \%$ strain reduction when compared for square and polygon respectively. This is due to different orientation of internal built structure which is also effects the collapsibility of patterns. Besides that, it was found that, there was a great difference in percentages of circumferential shell strain reduction which was $38 \%$. This is due to higher strain induced on the ceramic shell impact on the cracking behaviour of shell. However, in terms of axial shell strain, the reduction of strain found was $11 \%$ for square and polygon respectively. This behaviour can be understandable in terms of shell cracking, whereby, less strain happened to the shell considered to reduce the stress on the shell. By doing so, the shell can withstand the high pressure expansion of the pattern and will collapsed inwardly. Furthermore, the glass transition temperature was happened near $110^{\circ}$ $\mathrm{C}$ whereby it was an important characteristic of collapsibilty compared to MOR and CTE for determining the cracking behaviour of shell.

\section{Acknowledgement}

The authors wish to thank Ministry of Education Malaysia for funded Fundamental Research Grant Scheme(FRGS) Vot1423 and Additive Manufacturing Research Group(ADDMAN) at Faculty of Mechanical \& Manufacturing Engineering in Universiti Tun Hussein Onn Malaysia(UTHM) for facilitating and financial support to this research programme.

\section{References}

1.C. K. Chua, C. Feng, C. W. Lee, and G. Q. Ang, 'Rapid investment casting: Direct and indirect approaches via model maker II', Int. J. Adv. Manuf. Technol., vol. 25, no. 1-2, pp. 26-32, (2005)

2.Y. Ding, H. Lan, J. Hong, and D. Wu, 'An integrated manufacturing system for rapid tooling based on rapid prototyping', vol. 20, pp. 281-288, (2004).

3.C. W. Lee, C. K. Chua, C. M. Cheah, L. H. Tan, and C. Feng, 'Rapid investment casting: Direct and indirect approaches via fused deposition modelling', Int. J. Adv. Manuf.

Technol., vol. 23, no. 1-2, pp. 93-101, (2004)

4.A. Azari and S. Nikzad, 'The evolution of rapid prototyping in dentistry: a review', Rapid Prototyp. J., vol. 15, no. 3, pp. 216-225, (2009)

5.S. Jones and C. Yuan, 'Advances in shell moulding for investment casting', J. Mater. Process. Technol., vol. 135, no. 2-3 SPEC., pp. 258-265, (2003)

6.W. L. Yao and M. C. Leu, 'Analysis of shell cracking in investment casting with laser stereolithography patterns', Rapid Prototyp. J., vol. 5, no. 1, pp. 12-20, (1999)

7.W. L. Yao and M. C. Leu, 'Analysis and design of internal web structure of laser stereolithography patterns for investment casting', Mater. Des., vol. 21, no. 2, pp. 101$109,(2000)$

8.Y. Norouzi, S. Rahmati, and Y. Hojjat, 'A novel lattice structure for SL investment casting patterns', Rapid Prototyp. J., vol. 15, no. 4, pp. 255-263, (2009)

9.O. M. F. Marwah, S. Sharif, S. Sulaiman, M. Ibrahim, and E. J. Mohamad, 'Direct Investment Casting Numerical Study for ABS P400 FDM Materials', Appl. Mech. Mater., vol. 660, pp. 99-103, (2014)

10.W. S. W. Harun, S. Safian, and M. H. Idris, 'Evaluation of ABS patterns produced from FDM for investment casting process', WIT Trans. Eng. Sci., vol. 64, no. 3, pp. 319-328, (2009) 
11.O. Mohd, F. Marwah, S. Sharif, and M. Ibrahim, 'Direct Fabrication of IC Sacrificial Patterns via Rapid Prototyping Approaches', vol. 6, no. 5, (2012) 